\title{
Histometric and Histological Analysis of Bone to Screw Contact Between Metallic and Absorbable 2.0 Fixation System: A Pilot Study in Dogs
}

\section{Felipe Perraro Sehn}

Department of Oral and Maxillofacial Surgery and Periodontology, School of Dentistry of Ribeirão Preto, University of São Paulo, Brazil

\section{Rogério Kato}

Department of Oral and Maxillofacial Surgery and Periodontology, School of Dentistry of Ribeirão Preto, University of São Paulo, Brazil

\section{Paulo de Oliveira}

Department of Morphology, Stomatology and Physiology, School of Dentistry of Ribeirão Preto, University of São Paulo

\section{Alexandre Trivellato}

Department of Oral and Maxillofacial Surgery and Periodontology, School of Dentistry of Ribeirão Preto, University of São Paulo, Brazil

Cássio Sverzut ( $\square$ cesve@forp.usp.br)

Department of Oral and Maxillofacial Surgery and Periodontology, School of Dentistry of Ribeirão Preto, University of São Paulo, Brazil

\section{Research Article}

Keywords: Histometric, Histological, Metallic, Absorbable 2.0 Fixation

Posted Date: December 6th, 2021

DOI: https://doi.org/10.21203/rs.3.rs-1109075/v1

License: (c) (1) This work is licensed under a Creative Commons Attribution 4.0 International License. Read Full License 


\section{Abstract}

Objective

To evaluate the bone/screw interface of a titanium IF system and an absorbable IF system.

Materials and methods

Twelve male adult dogs were subjected to osteotomy performed between the third and fourth mandibular premolars, been divided into two groups (control or experimental side). Two miniplates were applied to fix mandible fragments. Histometric values for bone-to-screw contact (BSC), bone-to-screw distance (BSD) and histological findings were evaluated at 2 and 18 weeks.

Results

Difference in BSC were detected from absorbable and metallic monocortical group on the experimental side at 2 weeks $(p=0.0041)$. Larger BSDs were detected at buccal bicortical absorbable experimental sides $(p=0.0041)$, with larger distances detected from the vestibular side to the lingual cortical side $(p<$ $0.0001)$ and on the control side at 18 weeks $(p=0.0143)$, having metallic group significant BSD results on the experimental side at 2 weeks $(p=0.047)$. Connective tissue was noted on vestibular cortical side in monocortical and bicortical absorbable screw groups.

Conclusions

Absorbable bicortical screws with splint had histometric and histological behaviors similar to metallic bicortical group at lingual cortical side.

Clinical Relevance

Inion absorbable bicortical screws did not promote connective tissue formation at lingual cortical side.

\section{Introduction}

The use of metallic and absorbable internal fixation (IF) systems to treat fractures and osteotomies in the facial skeleton is well described in the literature, with indications and limitations for each case 1,2. Metallic systems made from titanium and its alloys are considered the "gold standard" for internal fixation (IF), promoting biomechanical stability, bone repair and an immediate return to function. Nevertheless, some problems have been associated with these systems, such as corrosion, imaging artifacts, allergic reactions, thermal sensitivity and osteoporosis or bone weakening by the stressshielding effect, leading surgeons to search for other types of IF systems ${ }^{3-8}$. Absorbable systems are related to reducing or eliminating these problems; additionally, they have the potential to eliminate an additional surgical procedure to remove the hardware, which is a routine procedure for metallic IF systems in some countries. Nevertheless, some controversies about absorbable systems in the 
scientific literature deserve further study, such as the type of material to be used and its constitution, the type of bone formation present in the fractured/osteotomized site, and the degradation process generated by the absorbable systems as well as nonrandomized and noncontrolled clinical studies in most cases ${ }^{9-11}$. Additionally, absorbable IF systems have also been associated with some disadvantages when compared to metallic systems, such as higher costs ${ }^{12}$, lower mechanical resistance and more time being required for the completion of the bone repair process ${ }^{13,14}$.

It is worth emphasizing that there is an important relationship between bone repair processes that occur simultaneously at fractured or osteotomized sites and at the bone/screw interface. The occurrence of the bone repair process at fractured or osteotomized sites depends directly on the stability between bone fragments provided by plates and screws. Furthermore, the retention of screws in bone tissue is essential for IF system stability and depends on the bone repair process at the bone/screw interface. While metallic IF systems do not alter their structural components during the bone repair process, absorbable IF systems must gradually transfer the stress function to bone while the IF system

simultaneously degrades ${ }^{15,16}$. Furthermore, various degradable polymers with distinct molecular weights, crystallinities, mechanical properties, and rates of degradation have been proposed. Therefore, the purpose of the present study was to evaluate the bone/screw interface of a titanium IF system and an absorbable IF system made with a combination of L-polylactic acid (LPLA), D, L-polylactic acid (DLPLA) and trimethylene carbonate (TMC) by histometric and histological analysis.

\section{Results}

\section{Histometric analysis}

The mean percentages and SDs of BSC for all groups are presented in Table 1. One-way ANOVA revealed statistically significant differences in BSC at 2 weeks from monocortical and bicortical metallic screws positioned in the same miniplate, with no statistically significant differences detected at 18 weeks and no significant differences detected in the absorbable groups at 2 and 18 weeks (Table 2). A statistically significant difference only was detected in the absorbable monocortical group compared to the metallic monocortical group on the experimental side at 2 weeks ( $p=0.0004$ - Fig. 1 ), with no statistically significant differences detected in the bicortical groups (Fig. 2). Larger BSDs were detected at the buccal bicortical absorbable experimental sides at both evaluated periods $(p=0.0041)$, with larger distances detected on the buccal cortical side when compared to the distances of the lingual cortical side from the same group $(p<0.0001)$ even on the control side at 18 weeks $(p=0.0143$ - Fig. 3$)$; in the metallic groups larger distances were observed only on the experimental side at 2 weeks $(p=0.047-$ Fig. 4).

\section{Histological analysis}

\section{2 weeks}


Monocortical absorbable screws: Soft connective tissue was noted adjacent to the surface of the monocortical absorbable screws, except for occasional areas showing newly formed woven bone trabeculae and the presence of parent, cortical lamellar bone juxtaposed to the first one or two threads underneath the head portion of the screw (Fig. 5A). The screws exhibited some cracks in their internal structure but no gaps in their surface.

Monocortical metallic screws: In addition to the histological characteristics of the connective tissues observed for the monocortical absorbable screws, the metallic screws had some areas between threads that were filled with amorphous bone debris (Fig. 5B) and pronounced osteoclastic activity in focal areas of the cancellous lamellar bone close to the threads in the medullary canal (Fig. 5B, inset). Some cracks in the parent cortical bone were seen adjacent to the metallic screw surface (Fig. 5B).

Bicortical absorbable screws: In both buccal and lingual cortical sides (Figs. $5 \mathrm{C}$ and E, respectively), the edges of the parent lamellar bone followed the contour of the screw surface, except in some interfacial areas that were filled with loose connective tissue. However, the parent bone matrix was only in direct contact with the screw surface in a few areas. Newly formed woven bone was detected adjacent to the screws in both the inner and outer cortical bone surfaces as a result of endosteal and periosteal reactions, respectively (Fig. $5 \mathrm{C}$ and E). Cracks in the screw structure were seen between its body and its head (Fig. 5C).

Bicortical metallic screws: In both buccal and lingual cortical sides (Figs. 5D and F, respectively), the edges of the pre-existing lamellar bone followed the contour of the screw surface, establishing direct contact with it, except in some focal areas that exhibited interposed loose connective tissue. Newly formed woven bone was detected adjacent to the screws, mostly associated with an endosteal reaction (Fig. 5D, inset). Bone debris remained in the bone marrow adjacent to the screw surface and the inner surface of the lingual cortical bone (Fig. 5F).

\section{8 weeks}

Monocortical absorbable screws: Although lamellar cortical and spongy bone surrounded the entire screw body surface following its contour, dense connective tissue was seen interposed between the bone and the screw. Only rarely have bone margins established direct contact with the screw surface. The cracks observed in the screw structure did not exhibit any formation of tissue ingrowth (Fig. 6A).

Monocortical metallic screws: Lamellar cortical and spongy bone surrounded the entire screw body, establishing direct contact with its surface, except in focal areas where loose connective tissue was noted (Fig. 6B).

Bicortical absorbable screws: Although both buccal and lingual cortical sides exhibited lamellar bone adjacent to the body of the screw (Figs. 6C and E, respectively), only the latter showed direct contact between the bone margins and the screw surface and showed a pronounced periosteal reaction. In the buccal cortex (Fig. 6C), a thin layer of dense connective tissue was formed between the bone matrix and 
the screw surface, extending to the bone marrow through the interfacial area up to the inner surface of the lingual cortex (Fig. 6E). The interfacial, dense connective tissue showed collagen fiber bundles in a multidirectional array that was highly vascularized. Multinucleated giant cells adhered to the irregular, degrading absorbable screw surface. The bone margin showed areas with an osteoid layer that was lined by osteoblasts (Fig. 6G). The cracks in the screw structure were mainly located in its core portion (Figs. $6 \mathrm{C}$ and $\mathrm{E})$.

Bicortical metallic screws: For both buccal and lingual cortical sides, the bone margins were in direct contact with the screw surface, except in a few areas where loose connective tissue was interposed between the bone and the screw surface (Figs. 6D and F). In bone marrow, a layer of dense connective tissue covered the screw surface, yet some threads were in direct contact with trabeculae of spongy bone (Figs. 6D and F) and occasionally showed active contact osteogenesis (Fig. 6H). A pronounced periosteal reaction was associated with the screw tip on the outer surface of the lingual cortex (Fig. 6F).

\section{Discussion}

It is essential to understand the relationship between the bone repair processes that occur simultaneously at the osteotomy or fracture site and at the interface between bone and screws. The bone repair process only occurs at the osteotomy or fracture site if the IF system provides adequate stability between bone fragments while the repair phenomenon occurs. Secure anchorage of osteosynthesis screws in bone is crucial for the uneventful healing of mandibular fractures and osteotomies in orthognathic surgery, particularly in the initial healing stages ${ }^{18}$. Theoretically, closer BSC leads to greater friction between the bone and screw, thus leading to better anchorage ${ }^{19,20}$. The present study used the same sample that was used in a previous in vivo study 14,21 in which the bone repair process at the osteotomy site was analyzed; however, the present study focused on assessing the interface between the bone and the screws by measuring the BSC and the BSD.

The results of BSC obtained in the previous study that was carried out with the same methodology were compared with the present study using different IF systems, with some distinct results achieved 1822 . Regarding the metallic IF system and the experimental side, the mean percentage of BSC at 2 weeks for the monocortical screws was $24.9 \% \pm 6.4 \%$, whereas in the present study, the mean percentage of BSC was $31.67 \% \pm 18.48 \%$. For the bicortical screws on the same side and period, the mean percentages of BSC were $32.2 \% \pm 15.1 \%$ and $6.56 \% \pm 3.17 \%$ for the previous and present studies, respectively. Analyzing the same side, a previous study found mean BSC percentages of $16.9 \% \pm 9.9 \%$ and $26 \% \pm 13.9 \%$ for mono- and bicortical screws, respectively, at 12 weeks. The present study found mean BSC percentages of $17.25 \% \pm 11.80 \%$ and $6.77 \% \pm 4.81 \%$ for mono- and bicortical screws, respectively, at 18 weeks.

Concerning the absorbable IF system and the experimental side, in a previous study ${ }^{22}$, the mean percentage of BSC at 2 weeks for monocortical screws was $4.93 \% \pm 8.92 \%$, whereas in the present study, it was $4.17 \% \pm 7.66 \%$. For the bicortical screws on the same side and period, the mean 
percentages of BSC were $4.18 \% \pm 3.02 \%$ and $4.00 \% \pm 3.51 \%$ for the previous and present studies, respectively. Analyzing the same side, a previous study found mean BSC percentages of $0.0 \% \pm 0.0 \%$ and $0.84 \% \pm 1.97 \%$ for mono- and bicortical screws, respectively, at 18 weeks. The present study found mean BSC percentages of $0.22 \% \pm 0.70 \%$ and $3.98 \% \pm 5.43 \%$ for mono- and bicortical screws, respectively, at 18 weeks.

For the metallic IF system only, statistically significant differences in BSC were observed between the screws positioned on the same plate at the 2-week period. This finding was noted on the experimental side for both mono- and bicortical screws, whereas for the control group, it was only observed for bicortical screws. Furthermore, greater BSDs were detected in monocortical screws and on the buccal cortical side of bicortical screws from the absorbable group at both evaluation points, and was even observed in the control group at 18 weeks; however, in the metallic group, larger BSDs were observed only on the experimental side at 2 weeks. Nonetheless, in a previous study analyzing a metallic IF system made in Brazil ${ }^{18}$, no significant difference was observed in BSC between monocortical and bicortical screws positioned in the same plate at each time point. Furthermore, no significant difference was observed between the experimental and control sides for either monocortical or bicortical screws. For the absorbable group, on the other hand, after the first period of evaluation, lower values were detected as time progressed, except in the bicortical group at 18 weeks, which showed greater values.

Regardless of the group, the overall findings could be due to an intense bone remodeling process occurring adjacent to the buccal screw surface, with resorption being predominant rather than apposition, most likely as a result of damage to bone and bone marrow tissues from drilling and screw insertion occurring at the first period of time ${ }^{18,22}$, material applied during the manufacturing process and the stability that the system had generated by the second period of evaluation.

In the present study, some cracks were noted in the screw body, similar to those observed in our previous study when a similar absorbable system was analyzed ${ }^{22}$. Nonetheless, the cracks noticed at 18 weeks in the present study were empty, and some of those from our previous study were filled with loose connective tissue with aggregates of inflammatory cells, with focal areas of multinucleated giant cells. In the present study, we did not observe bulk resorption but instead noted multinucleated giant cells at the hither screw surface, with the surface of the threads intact, even when the internal axis was full of cracks. Furthermore, we did not observe connections between the cracks present at the surface of the threads and those in the internal axis of the screws. This result is not in accordance with our previous study in which the cracks and gaps were fulfilled with tissue similar to that observed surrounding the screws ${ }^{22}$. Furthermore, this difference resulted from the material applied to manufacture each IF system once the management of screws and plates was the same. It is worth suggesting that at 18 weeks, the screws in our previous study ${ }^{22}$ were in a more advanced stage of resorption than those in the present study. Eglin and Alini ${ }^{23}$ suggested that the cracks and gaps could be attributed to both the penetration of molecules of water through the amorphous part of the material and the occurrence of hydrolysis by scissions of the ester bond, reducing the $\mathrm{pH}$ level in tandem with an 
increasing rate of hydrolytic scission of the screw. Riemann, et al. ${ }^{24}$ showed that an acidic environment increased the expression of inflammatory markers such as TNF-a, COX-2 and iNOS in fibroblasts. These markers are known to be effective inhibitors of osteoblasts ${ }^{25-27}$, especially at higher concentrations of iNOS, which can explain why large amounts of collagen fiber bundles were found with osteoblasts at distances from absorbable screws, with fibroblasts and osteoblasts being responsible for collagen fiber synthesis ${ }^{28,29}$. According to Hochuli-Vieira, et al. ${ }^{30}$, fibrous connective tissue was found proportional to the study time, meaning that the longer the evaluation period was, the smaller the amount of bone around the screws and the larger the connective tissue was. Congruous with this, our study detected that the farther the long axis of the screw is from the IF instability source, the larger the BSC is, even at the control side, at 18 weeks. Histologically, this behavior occurred not only by bone absence resorption surrounding the screw at the lingual cortical side but also for endosteal and periosteal bone formation at this cortical side. Although Reitzik and Schoorl ${ }^{31}$ and Rasubala, et al. ${ }^{32}$ explained that a periosteal reaction could be due to interfragmentary movements, the absence of fragments in the bicortical absorbable group from our study could not be linked to such an occurrence. New bone formation without any sign of osteolysis on the lingual cortical side could be an effect of periosteum detachment and screw stability, promoting a greater amount of BSC at bicortical absorbable screws as well as at bicortical metallic screws on the lingual cortical side.

We detected that inflammatory capsules were present only on the buccal cortical side of the absorbable screw surface, meaning that not only was the biomaterial constitution essential to promote fibrous capsule growth but also to promote the instability of the system at that portion, with multinucleated giant cells resorbing absorbable screws. Bat, et al. ${ }^{33}$ showed that films of poly (trimethylene carbonate) were only eroded when macrophages were cultured directly on the film, which was required to resorb the absorbable screw surface. Our histological findings suggested that the absorbable system screw resorption process was initiated by the inner surface by multinucleated giant cells. Dondossola, et al. ${ }^{34}$ observed that M1 macrophage phenotype cells became immobilized along the scaffold/tissue interface, forming multinucleated giant cells; both M1 macrophage phenotype cells and multinucleated giant cells produced vascular endothelial growth factor (VEGF), initiating and maintaining an immature neovessel network, followed by the formation of a dense collagen capsule two to four weeks after implantation. Furthermore, Yu, et al. ${ }^{35}$ established that macrophage polarization into the M2 phenotype was also responsible for fibrous capsule thickness; in addition to the fact that the M2 phenotype is important for angiogenesis ${ }^{36}$, the M1 and M2 phenotypes have been found in foreign body reactions ${ }^{37}$.

The histometric and histological findings support that the metallic IF system provoked fewer tissue reactions than the absorbable group and generated a stable environment. It is worth noting that contact osteogenesis was detected at the metallic interface at 18 weeks, created by appropriate surface topography and metallic alloys ${ }^{38}$ with new bone formation from the screw surface to the host bone ${ }^{39}$. This can be considered an advantage in most cases; however, it can be a disadvantage in those cases in which the metallic IF system needs to be removed for a specific reason. 
Regardless of the group (metallic and absorbable), the histological analysis of the present study did not reveal any differences between the control and experimental sides, showing similar findings on both sides. Thus, it is worth noting that the IF systems (plus the dental splint in the absorbable group) were capable of stabilizing bone fragments, thus neutralizing the forces generated by muscle actions.

Although the histological and histometric findings at the BSC interface between the metallic and absorbable IF systems were distinct, both were capable of providing conditions needed for the bone repair process to occur at the osteotomy site, as observed in our previous studies with the same sample. Additionally, the patterns of repair were clearly distinct, and the compression third was always in a more advanced stage of bone repair than the tension third as a consequence of biomechanical behavior ${ }^{14}$ [22].

The goal of this study and of previous studies ${ }^{14} 18$ [22] was to understand the relationship and correlation between the bone repair processes that occur simultaneously at the osteotomy/fracture site and at the interface between screws and bone in both metallic and absorbable systems. We used the metallic system as a control group because this system has been successfully used for a longer time than absorbable systems. This comparison was conducted to understand the bone repair process in each specific IF system and not to determine if one is superior to another. Furthermore, each IF system has specific indications, and in most cases, there are no conflicting issues about this matter. In conclusion, each IF system has a distinct pattern of bone repair processes at the BSC interface resulting from the material used when the screw was manufactured, the protocol used for screw insertion and the biomechanical behavior generated by the IF system itself.

\section{Materials And Methods}

This study protocol was reviewed and approved by the Institutional Animal Care and Use Committee of the University of São Paulo (process 07.1.188.53.9), and the same veterinarian conducted all animal management at Experimental Animal Care Facility II. Twelve healthy male adult mongrel dogs aged between 3 and 8 years old weighing from 15 to $20 \mathrm{~kg}$ were used as subjects. General anesthesia was achieved by intravenous administration of zolazepam/tiletamine $(0.12 \mathrm{ml} / \mathrm{kg})$, which was maintained via isoflurane and oxygen inhalation through an orotracheal tube. The randomization was realized by sorting the dogs into two groups after a simple random selection to determine which IF system would be applied (absorbable and metallic groups) and which side would be the control or experimental side. Afterward, on the experimental side, an iatrogenic osteotomy was performed between the third and fourth mandibular premolars through an intraoral approach using a surgical microreciprocating saw with a surgical blade that was $0.2 \mathrm{~mm}$ thick. The surgical blade was positioned perpendicular to the mandibular body, and a continuous defect was created. Two perforations were made at the mandibular base near the osteotomy in both fragments so that $155 \mathrm{~mm}$ self-lock reduction forceps (Synthes, Oberdorf, Switzerland) could be used to reduce and stabilize the fragments. The contralateral side was determined as the control side, and the same surgical approach was applied. Nonetheless, the vestibular cortical bone between the third and fourth premolars was only slightly osteotomized; thus, no bone discontinuity was produced. 


\section{Metallic Group}

The IF system was applied according to the Association for the Study of Osteosynthesis - AO recommendation for a transversal fracture line without dislocation ${ }^{17}$. A $2.0 \mathrm{~mm}$ nonlocking system (Synthes, Oberdorf, Switzerland) was applied, and two 4-hole titanium plates were fixed with 4 titanium alloy self-tapping screws each. Two drills with a diameter of $1.5 \mathrm{~mm}$ were used to drill the holes, one with a stop length of $6.0 \mathrm{~mm}$ near the alveolar process and another with a stop length of $20.0 \mathrm{~mm}$ near the mandibular base. The plate positioned near the alveolar process was fixed monocortically with $6.0 \mathrm{~mm}$ self-tapping screws, and the plate near the mandibular base was fixed bicortically. To ensure bicortical engagement, the length of the screws was selected according to the vestibular-lingual dimension of the mandible, which varied from 8.0 to $12.0 \mathrm{~mm}$.

\section{Absorbable Group}

The $2.0 \mathrm{~mm}$ IF system used was manufactured with the combination of L-polylactic acid (LPLA), D, Lpolylactic acid (DLPLA) and trimethylene carbonate (TMC), following the manufacturer's recommendations (Inion, Tampere, Finland). Accordingly, with the same design as the Metallic Group, two 4-hole plates were fixed by applying 4 nonlocking screws in each plate. To achieve adequate adaptation to buccal cortical tissue, the plates were immersed in sterile saline solution warmed to $55^{\circ} \mathrm{C}$ in a water bath (Thermo, Inion, Tampere, Finland) for 5 minutes and were allowed to adapt to the bone surface via digital manipulation. Two drill bits with diameters of $1.75 \mathrm{~mm}$ and stop lengths of $6.0 \mathrm{~mm}$ and $22.0 \mathrm{~mm}$ were applied; the first was used to generate holes near the alveolar process and the latter was used for holes near the mandibular base. Afterward, a tap was manually inserted to the full drill depth to provide threading for the screws. The plate positioned along the alveolar process was first fixed monocortically with screws $7.0 \mathrm{~mm}$ long, and then the second plate was fixed bicortically along the mandibular base. To ensure bicortical engagement, the length of the screws was selected according to the buccal-lingual dimension of the mandibular base, which varied from 9.0 to $13.0 \mathrm{~mm}$. Afterward, only for this group, a dental splint was created by applying a $0.8 \mathrm{~mm}$ orthodontic wire and light-cured composite resin involving the lingual surface of the first molar and buccal surfaces of the second, third and fourth premolars, as well as the canine teeth. The final configuration of the dental splint did not generate any form of occlusion interference during mandibular movements.

\section{Control Side}

In each specific group, the contralateral side was used as a control, and the same surgical approach and screw engagement procedures were performed. Nonetheless, a surgical blade was applied only to make a slight demarcation line on the buccal cortex to allow positioning of the IF system at the same place as that on the experimental side. Therefore, no bone discontinuity was created on the control side. Furthermore, only half of the IF system was used (one 2-hole plate fixed with 2 monocortical screws near the alveolar process and another 2-hole plate fixed with 2 bicortical screws along the mandible). 
Regardless of the group, after finishing the placement of IF systems, the surgical wound was copiously irrigated with sterile saline solution and closed with a 4-0 absorbable thread (Polyglactin 910, Ethicon/Johnson \& Johnson Medical N.V., Belgium) in a continuous fashion. Furthermore, no functional restriction was established, and all animals were placed on a diet of regular chow (dry granulated fodder) immediately after the surgical procedure. During the postoperative period, animals were randomly selected for evaluation at 2 and 18 weeks, and 3 dogs were killed per time period. The mandibular body was resected near the plate extremities and immersed immediately in $10 \%$ neutral buffered formalin solution. After fixation, the specimens from both sides (control and experimental) were dehydrated in an ascending series of ethyl alcohol and then infiltrated with methyl methacrylate. The hardened blocks were positioned in a microtome (Exakt Apparatebau GmbH \& Co, Norderstedt, Germany) and sectioned along the long axis of each screw, creating slices that were approximately $30 \mu \mathrm{m}$ thick. A combination of Stevenel's blue and Alizarin red was used to stain each slice for light microscopy observation.

\section{Histometric and Histological Analysis}

Histometric analysis was performed by applying a conventional and polarized light microscope (Leica Microsystems, Hesse, Germany) connected to a computer using a Leica DC300F digital camera (Leica Microsystems) for histometric readings, employing an objective lens of $2.5 \times$ magnification. Nonetheless, when more accuracy was needed, a lens of greater magnification was used. Excluding the head, each screw's perimeter was quantified, and the bone-to-screw contact (BSC) was expressed as a percentage. The bone-to-screw distance (BSD) was measured from the tops of 3 threads nearest to the bone surface on each side of the screw in one segment (buccal) for monocortical screws and in two segments (buccal and lingual) for bicortical screws. Histological analysis was performed in the same apparatus as was used for the histometric evaluation. The interface between the monocortical screws and bone was evaluated in two segments (buccal and medullary), whereas the bicortical screw was evaluated in three segments (vestibular, medullary and lingual).

\section{Statistical Analysis}

Mean values (\%) and standard deviation (SD) results were calculated for bone-to-screw contact (BSC sum of buccal, medullar and lingual) and for bone-to-screw distance (BSD). After applying the ShapiroWilk normality test for histometric data and one-way analysis of variance (ANOVA) with Dunn's post hoc correction test, a significance level of 0.05 was used. When data was shown to be nonparametric, the Kruskal-Wallis test was applied for multiple comparisons. Statistical data were analyzed with GraphPad Prism software, version 8.1.1 (330) RRID:SCR_002798 (GraphPad Software Inc., San Diego, United States of America).

\section{Declarations}

\section{Author Contributions}


All authors meet the criteria for authorship and all those entitled to authorship are listed as authors. CES and AET designed the study on methodological properties and ensured approval of the Ethical Committee. Animal surgeries were done by RBK, AET, CES and FPS carried out all histometric measurements, histological acquirements and statistical analysis. FPS, PTO and CES analyzed all data provided and wrote the study. All authors read and approved the final manuscript.

\section{Funding}

This study was part of a research funded by São Paulo Research Foundation (FAPESP Grants No. 2007/00892-8 and 2014/23710-6) and by the Coordenação de Aperfeiçoamento de Pessoal de Nível Superior - Brasil (CAPES) - Finance Code 001.

\section{Competing interests}

The authors declare no competing interests.

\section{Ethical approval}

"This is to certify that the work (Proc. no. 2007.1.188.53.9), entitled 'Histometric and histological analysis of bone-to-screw contact between metallic and absorbable 2.0 fixation systems: a pilot study in dogs', by Cássio Edvard Sverzut, is in accordance with the Ethical Principles in Animal Experimentation adopted by Ethical Committee on Animal Experimentation (CEUA) of the campus of Ribeirão Preto - USP (PUSP-RP) and was approved in the meeting on May $08^{\text {th }}, 2007 . "$

Authors confirm that this study is reported in accordance with ARRIVE guidelines.

\section{References}

1 Kumar, A. V., Staffenberg, D. A., Petronio, J. A. \& Wood, R. J. Bioabsorbable plates and screws in pediatric craniofacial surgery: a review of 22 cases. The Journal of craniofacial surgery 8, 97-99 (1997).

2 Yang, L. et al. Skeletal stability of bioresorbable fixation in orthognathic surgery: a systemic review. Journal of cranio-maxillo-facial surgery : official publication of the European Association for Cranio-Maxillo-Facial Surgery 42, e176-181, doi:10.1016/j.jcms.2013.08.003 (2014).

3 Quereshy, F. A., Goldstein, J. A., Goldberg, J. S. \& Beg, Z. The efficacy of bioresorbable fixation in the repair of mandibular fractures: an animal study. Journal of oral and maxillofacial surgery : official journal of the American Association of Oral and Maxillofacial Surgeons 58, 1263-1269, doi:10.1053/joms.2000.16627 (2000).

$4 \quad$ Bahr, W., Stricker, A., Gutwald, R. \& Wellens, E. Biodegradable osteosynthesis material for stabilization of midface fractures: experimental investigation in sheep. Journal of cranio-maxillo-facial 
surgery : official publication of the European Association for Cranio-Maxillo-Facial Surgery 27, 51-57 (1999).

5 Pietrzak, W. S. Principles of development and use of absorbable internal fixation. Tissue engineering 6, 425-433, doi:10.1089/107632700418128 (2000).

6 Cabrini Gabrielli, M. A., Real Gabrielli, M. F., Marcantonio, E. \& Hochuli-Vieira, E. Fixation of mandibular fractures with 2.0-mm miniplates: review of 191 cases. Journal of oral and maxillofacial surgery : official journal of the American Association of Oral and Maxillofacial Surgeons 61, 430-436, doi:10.1053/joms.2003.50083 (2003).

7 Suzuki, T., Kawamura, H., Kasahara, T. \& Nagasaka, H. Resorbable poly-L-lactide plates and screws for the treatment of mandibular condylar process fractures: a clinical and radiologic follow-up study. Journal of oral and maxillofacial surgery : official journal of the American Association of Oral and Maxillofacial Surgeons 62, 919-924 (2004).

8 Chacon, G. E., Dillard, F. M., Clelland, N. \& Rashid, R. Comparison of strains produced by titanium and poly D, L-lactide Acid plating systems to in vitro forces. Journal of oral and maxillofacial surgery : official journal of the American Association of Oral and Maxillofacial Surgeons 63, 968-972 (2005).

9 Agarwal, S., Gupta, A., Grevious, M. \& Reid, R. R. Use of resorbable implants for mandibular fixation: a systematic review. The Journal of craniofacial surgery 20, 331-339, doi:10.1097/SCS.0b013e31819922fb (2009).

10 Dorri, M., Nasser, M. \& Oliver, R. Resorbable versus titanium plates for facial fractures. The Cochrane database of systematic reviews, CD007158, doi:10.1002/14651858.CD007158.pub2 (2009).

11 Yang, L. et al. Complications of absorbable fixation in maxillofacial surgery: a meta-analysis. PloS one 8, e67449, doi:10.1371/journal.pone.0067449 (2013).

12 van Bakelen, N. B. et al. Cost-Effectiveness of a Biodegradable Compared to a Titanium Fixation System in Maxillofacial Surgery: A Multicenter Randomized Controlled Trial. PLoS One 10, e0130330, doi:10.1371/journal.pone.0130330 (2015).

13 Buijs, G. J. et al. A randomized clinical trial of biodegradable and titanium fixation systems in maxillofacial surgery. J Dent Res 91, 299-304, doi:10.1177/0022034511434353 (2012).

14 Sverzut, C. E. et al. Comparative study of bone repair in mandibular body osteotomy between metallic and absorbable $2.0 \mathrm{~mm}$ internal fixation systems. Histological and histometric analysis in dogs: a pilot study. International journal of oral and maxillofacial surgery 41, 1361-1368, doi:10.1016/j.ijom.2012.04.012 (2012).

15 Martin, C., Winet, H. \& Bao, J. Y. Acidity near eroding polylactide-polyglycolide in vitro and in vivo in rabbit tibial bone chambers. Biomaterials 17, 2373-2380 (1996). 
16 Bergsma, J. E., de Bruijn, W. C., Rozema, F. R., Bos, R. R. \& Boering, G. Late degradation tissue response to poly(L-lactide) bone plates and screws. Biomaterials 16, 25-31 (1995).

17 Assael, L. A. Manual of Internal Fixation in the Cranio-Facial Skeleton Techniques Recommended by the AO/ASIF Maxillofacial Group. (Springer Berlin Heidelberg, 1998).

18 dos Santos, T. I. et al. Histological and histomorphometric analysis of the bone-screw interface in the mandibular body after using a 2.0-mm miniplate system: an experimental study in dogs. Journal of oral and maxillofacial surgery : official journal of the American Association of Oral and Maxillofacial Surgeons 65, 2169-2175, doi:10.1016/j.joms.2006.11.020 (2007).

19 Bähr, W. Comparison of torque measurements between cortical screws and emergency replacement screws in the cadaver mandible. J Oral Maxillofac Surg 50, 46-49 (1992).

20 Bähr, W. \& Lessing, R. The response of midfacial bone in sheep to loaded osteosynthesis screws in pretapped and nontapped implant sites. J Oral Maxillofac Surg 50, 1289-1294 (1992).

21 Sverzut, C. E. et al. Bone repair in mandibular body osteotomy after using 2.0 miniplate systemhistological and histometric analysis in dogs. International journal of experimental pathology 89, 91-97, doi:10.1111/j.1365-2613.2007.00569.x (2008).

22 Sverzut, C. E. et al. Histologic and Histometric Analysis of Bone Repair at the Site of Mandibular Body Osteotomy and at the Bone-Screw Interface After Using a Biodegradable 2.0-mm Internal Fixation System. The Journal of craniofacial surgery 26, 1214-1219, doi:10.1097/SCS.0000000000001723 (2015).

23 Eglin, D. \& Alini, M. Degradable polymeric materials for osteosynthesis: tutorial. European cells \& materials 16, 80-91, doi:10.22203/ecm.v016a09 (2008).

24 Riemann, A. et al. Acidic environment activates inflammatory programs in fibroblasts via a cAMP-MAPK pathway. Biochimica et biophysica acta 1853, 299-307, doi:10.1016/j.bbamcr.2014.11.022 (2015).

25 Yoon, D. S. et al. The effects of COX-2 inhibitor during osteogenic differentiation of bone marrowderived human mesenchymal stem cells. Stem cells and development 19, 1523-1533, doi:10.1089/scd.2009.0393 (2010).

26 Gilbert, L. et al. Inhibition of osteoblast differentiation by tumor necrosis factor-alpha. Endocrinology 141, 3956-3964, doi:10.1210/endo.141.11.7739 (2000).

27 van't Hof, R. J. \& Ralston, S. H. Nitric oxide and bone. Immunology 103, 255-261, doi:10.1046/j.1365-2567.2001.01261.x (2001). 
Bosetti, M., Zanardi, L., Hench, L. \& Cannas, M. Type I collagen production by osteoblast-like cells cultured in contact with different bioactive glasses. Journal of biomedical materials research. Part A 64, 189-195, doi:10.1002/jbm.a.10415 (2003).

29 Yonekura, S., Nagao, T. \& Arimori, S. Increased growth and collagen synthesis of bone marrow fibroblasts from patients with chronic myelocytic leukaemia. British journal of haematology 61, 93-99, doi:10.1111/j.1365-2141.1985.tb04064.x (1985).

30 Hochuli-Vieira, E., Cabrini Gabrielli, M. A., Pereira-Filho, V. A., Gabrielli, M. F. \& Padilha, J. G. Rigid internal fixation with titanium versus bioresorbable miniplates in the repair of mandibular fractures in rabbits. International journal of oral and maxillofacial surgery 34, 167-173, doi:10.1016/j.ijom.2004.03.016 (2005).

31 Reitzik, M. \& Schoorl, W. Bone repair in the mandible: a histologic and biometric comparison between rigid and semirigid fixation. Journal of oral and maxillofacial surgery : official journal of the American Association of Oral and Maxillofacial Surgeons 41, 215-218, doi:10.1016/0278-2391(83)90263$\mathrm{x}(1983)$.

32 Rasubala, L. et al. Comparison of the healing process in plated and non-plated fractures of the mandible in rats. The British journal of oral \& maxillofacial surgery 42, 315-322, doi:10.1016/j.bjoms.2004.02.020 (2004).

33 Bat, E., van Kooten, T. G., Feijen, J. \& Grijpma, D. W. Macrophage-mediated erosion of gamma irradiated poly(trimethylene carbonate) films. Biomaterials 30, 3652-3661, doi:10.1016/j.biomaterials.2009.03.033 (2009).

34 Dondossola, E. et al. Examination of the foreign body response to biomaterials by nonlinear intravital microscopy. Nature biomedical engineering 1, doi:10.1038/s41551-016-0007 (2016).

$35 \mathrm{Yu}, \mathrm{T}$. et al. Temporal and spatial distribution of macrophage phenotype markers in the foreign body response to glutaraldehyde-crosslinked gelatin hydrogels. Journal of biomaterials science. Polymer edition 27, 721-742, doi:10.1080/09205063.2016.1155881 (2016).

36 Spiller, K. L. et al. The role of macrophage phenotype in vascularization of tissue engineering scaffolds. Biomaterials 35, 4477-4488, doi:10.1016/j.biomaterials.2014.02.012 (2014).

37 Moore, L. B. \& Kyriakides, T. R. Molecular Characterization of Macrophage-Biomaterial Interactions. Advances in experimental medicine and biology 865, 109-122, doi:10.1007/978-3-31918603-0_7 (2015).

38 Choi, J. Y., Albrektsson, T., Jeon, Y. J. \& Yeo, I. L. Osteogenic Cell Behavior on Titanium Surfaces in Hard Tissue. Journal of clinical medicine 8, doi:10.3390/jcm8050604 (2019). 
39 Marco, F., Milena, F., Gianluca, G. \& Vittoria, O. Peri-implant osteogenesis in health and osteoporosis. Micron 36, 630-644, doi:10.1016/j.micron.2005.07.008 (2005).

\section{Tables}

Table 1: BSC values for each screw material applied, cortical system, side and study period.

\begin{tabular}{lll} 
Groups and weeks & Mean (\%) & SD (\%) \\
\hline Abs mono exp 2 & 2,39 & 4,22 \\
\hline Abs mono exp 18 & 0,30 & 0,86 \\
\hline Abs mono ctrl 2 & 7,43 & 11,51 \\
\hline Abs mono ctrl 18 & 0,08 & 0,20 \\
\hline Abs bicort exp 2 & 10,30 & 5,95 \\
\hline Abs bicort exp 18 & 6,66 & 4,94 \\
\hline Abs bicort ctrl 2 & 7,65 & 3,27 \\
\hline Abs bicort ctrl 18 & 10,32 & 6,91 \\
\hline Met mono exp 2 & 33,63 & 18,21 \\
\hline Met mono exp 18 & 16,23 & 13,36 \\
\hline Met mono ctrl 2 & 31,39 & 21,81 \\
\hline Met mono ctrl 18 & 24,63 & 13,89 \\
\hline Met bicort exp 2 & 13,91 & 4,94 \\
\hline Met bicort exp 18 & 13,95 & 9,53 \\
\hline Met bicort ctrl 2 & 14,59 & 1,68 \\
\hline Met bicort ctrl 18 & 13,35 & 4,94 \\
\hline
\end{tabular}

Table 2: Mean values for BSC of screws placed at the same plate and differences detected between them according to side of experiment/period and statistical differences. 
Experimental

Control

\begin{tabular}{llllllll} 
Week & Proximal & Distal & df & $P$ & Proximal & df & $P$ \\
\hline $\begin{array}{l}\text { Absorbable } \\
\text { monocortical screws }\end{array}$ & & & & & & & \\
\hline 2 & 1.54 & 3.11 & 9 & .55 & 7.43 & 6 & .27 \\
\hline 18 & 0.57 & 0.07 & 4 & .43 & 0.08 & 4 & .44
\end{tabular}

Absorbable bicortical screws

\begin{tabular}{llllllll}
\hline 2 & 9.09 & 11.11 & 8 & .60 & 7.65 & 5 & .62 \\
\hline 18 & 6.18 & 7.06 & 9 & .78 & 10.32 & 9 & .27 \\
\hline $\begin{array}{l}\text { Metallic monocortical } \\
\text { screws }\end{array}$ & & & & & & & \\
\hline 2 & 21.11 & 44.07 & 8 & .03 & 31.39 & 9 & .37 \\
\hline 18 & 14.81 & 17.45 & 11 & .73 & 24.63 & 10 & .23 \\
\hline $\begin{array}{l}\text { Metallic bicortical } \\
\text { screws }\end{array}$ & & & & & & & \\
\hline 2 & 10.71 & 17.11 & 10 & .01 & 14.59 & 7 & .04 \\
\hline 18 & 12.11 & 15.48 & 7 & .60 & 13.35 & 5 & .83
\end{tabular}

Figures 


\section{Monocortical Screws}

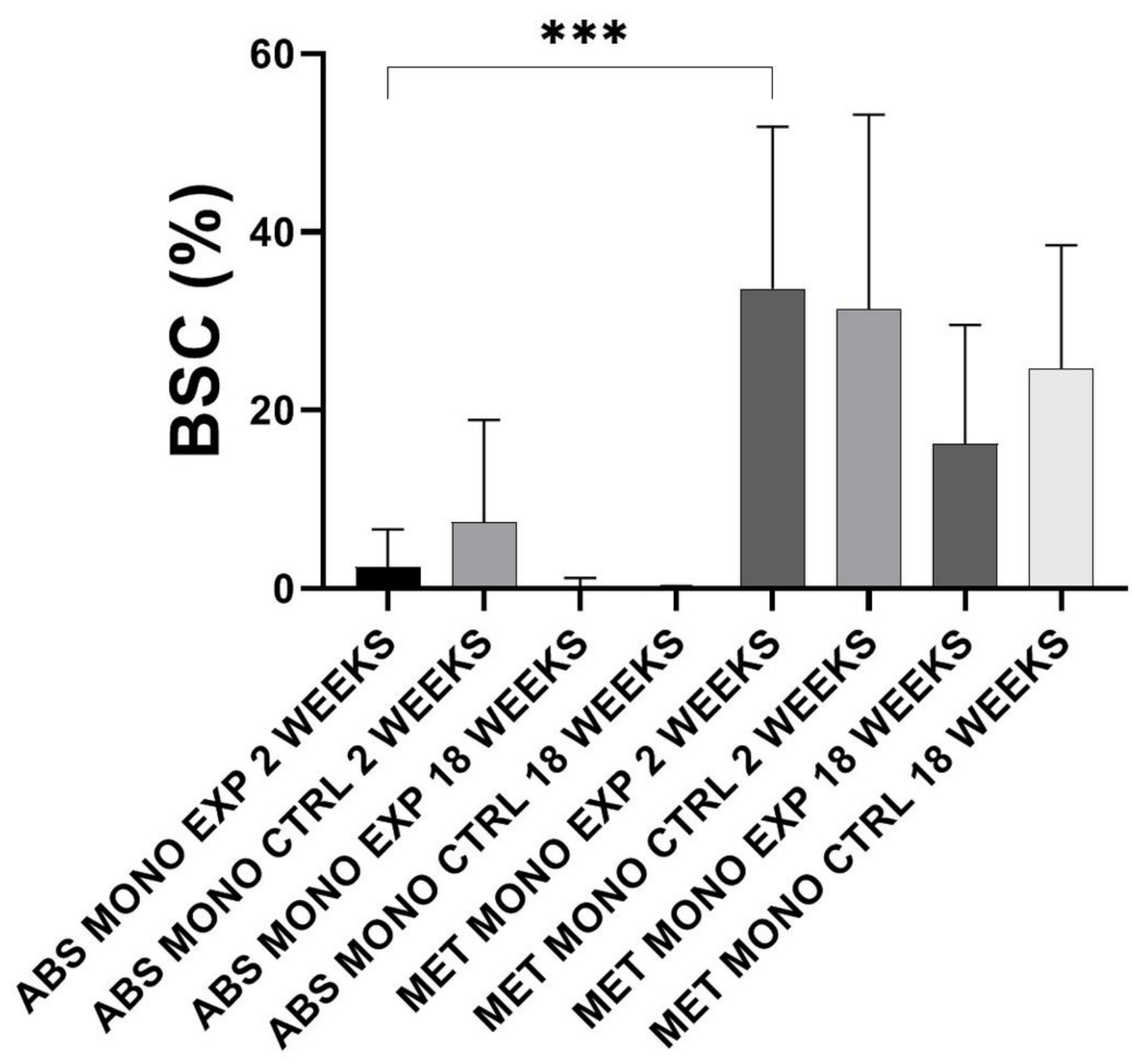

Figure 1

Bone to screw contact (BSC) percentage for monocortical screws. The data are presented as mean and standard deviation. The asterisks indicate statistically significant differences $(p=0.0004)$. Legends: absorbable (ABS); metallic (MET); monocortical (MONO); experimental (EXP); control (CTRL) 


\section{Bicortical Screws}

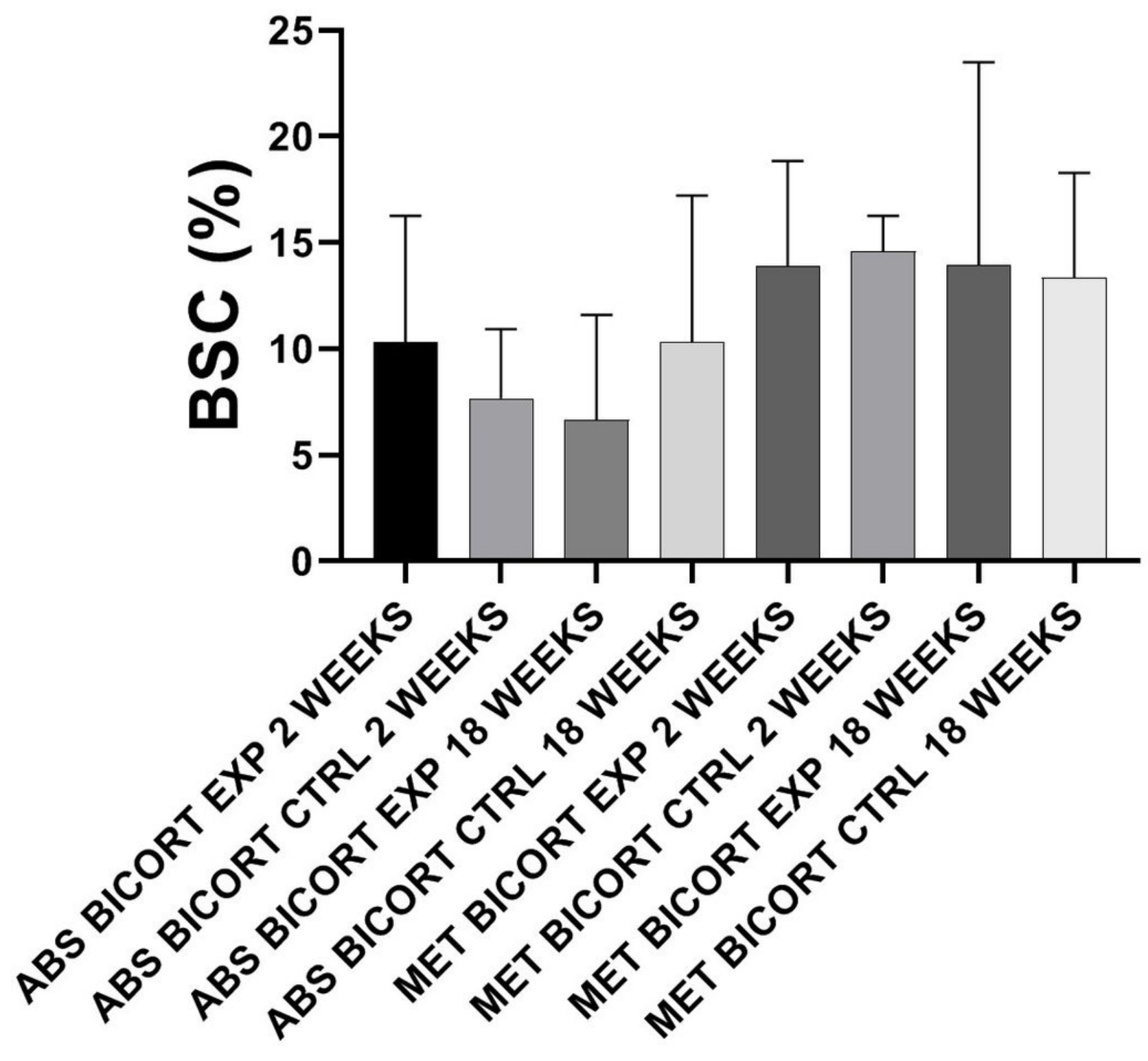

Figure 2

Bone to screw contact (BSC) percentage for bicortical screws. The data are presented as mean and standard deviation. No statistically significant differences are noted. Legends: absorbable (ABS); metallic (MET); bicortical (BICORT); experimental (EXP); control (CTRL) 


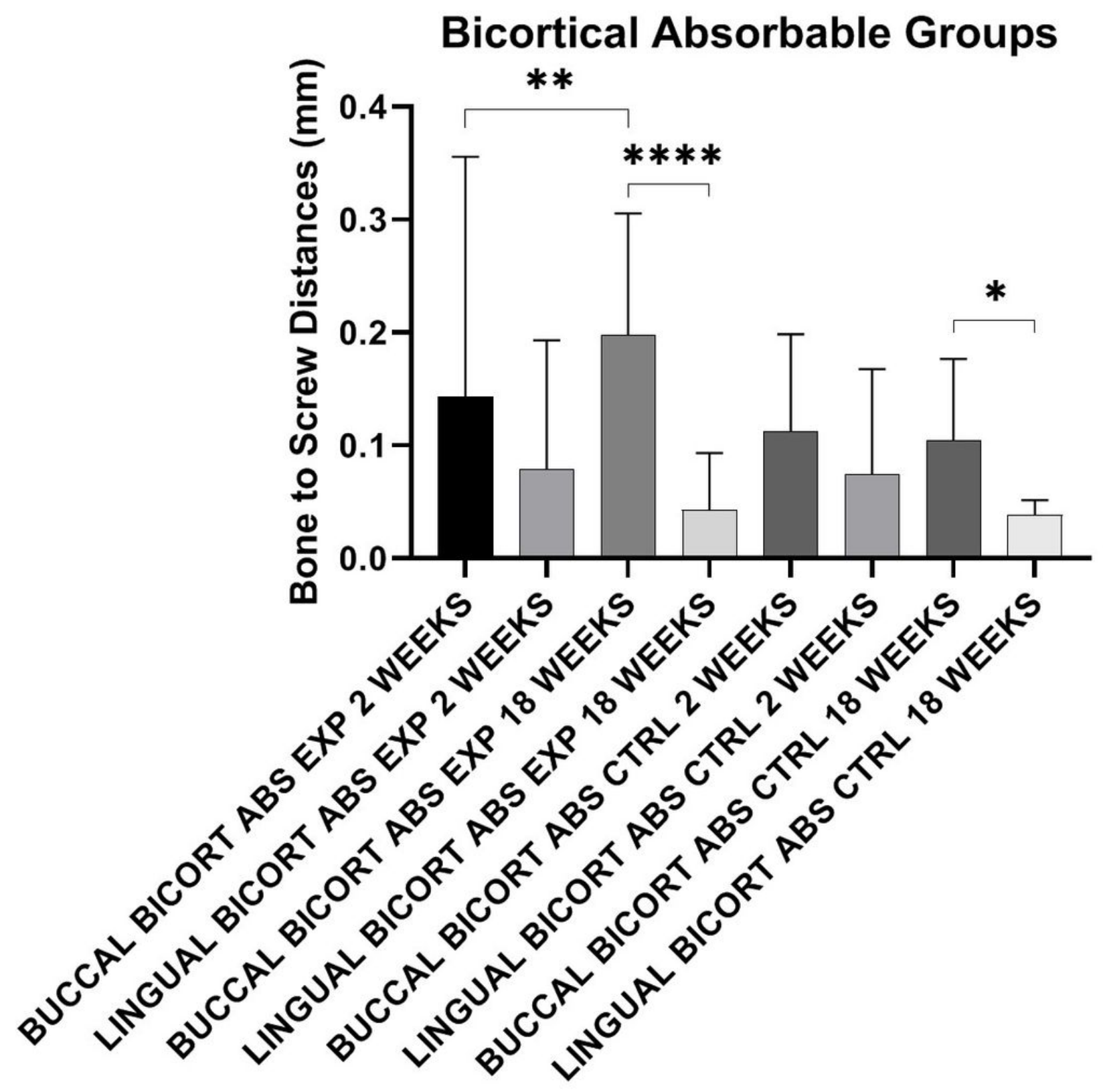

Figure 3

Bone to screw distances $(\mathrm{mm})$ for bicortical absorbable groups. The data are presented as mean and standard deviation. The asterisks indicate statistically significant differences: $* \star(p=0.0041)$; $* \star \star \star$ $(p<0.0001) ; *(p=0.0143)$. Legends: absorbable (ABS); bicortical (BICORT); experimental (EXP); control (CTRL) 


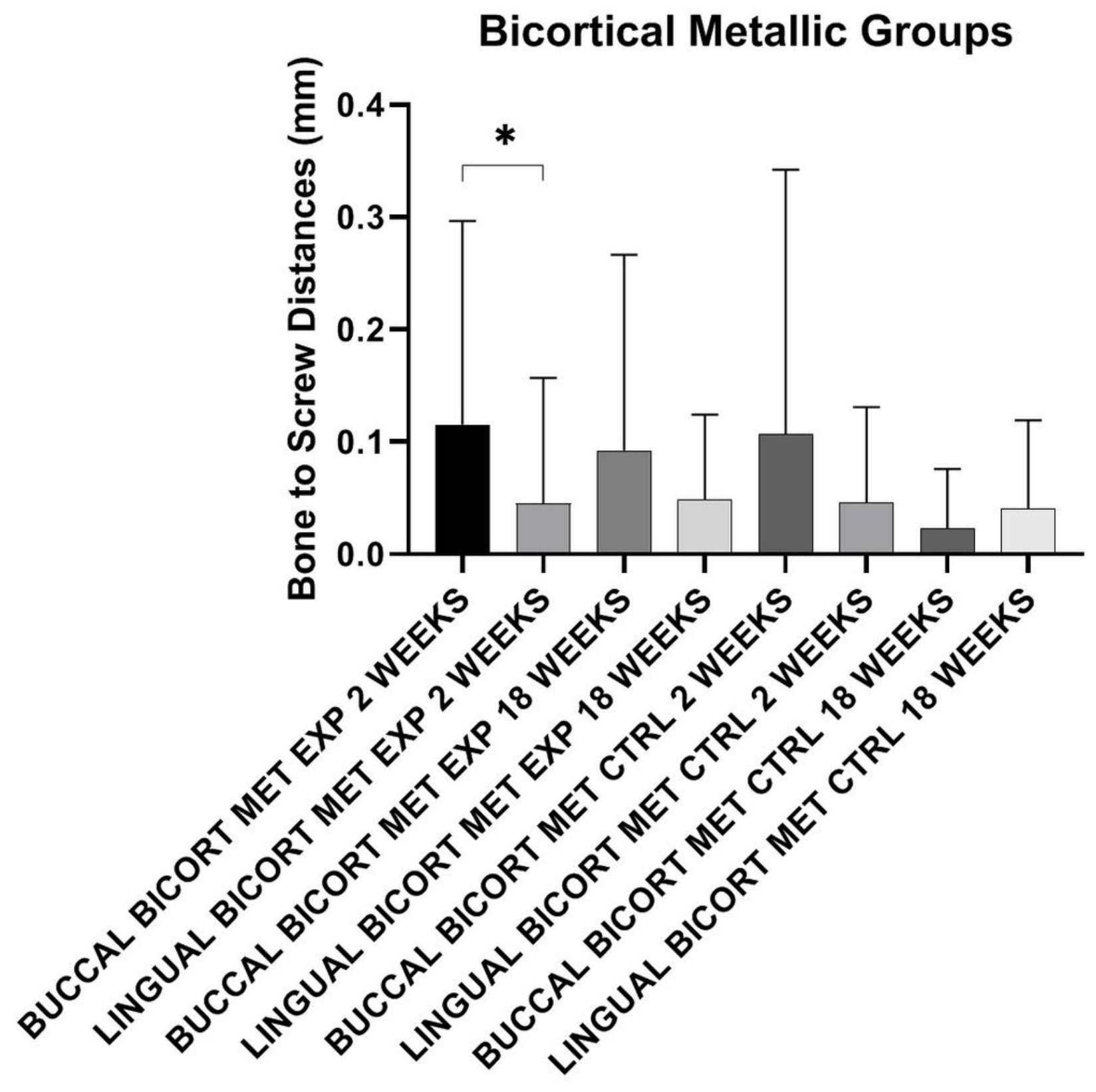

Figure 4

Bone to screw distances $(\mathrm{mm})$ for bicortical metallic groups. The data are presented as mean and standard deviation. The asterisk indicates statistically significant difference $(p=0.047)$. Legends: metallic (MET); bicortical (BICORT); experimental (EXP); control (CTRL) 


\section{2 weeks}

\section{Absorbable}
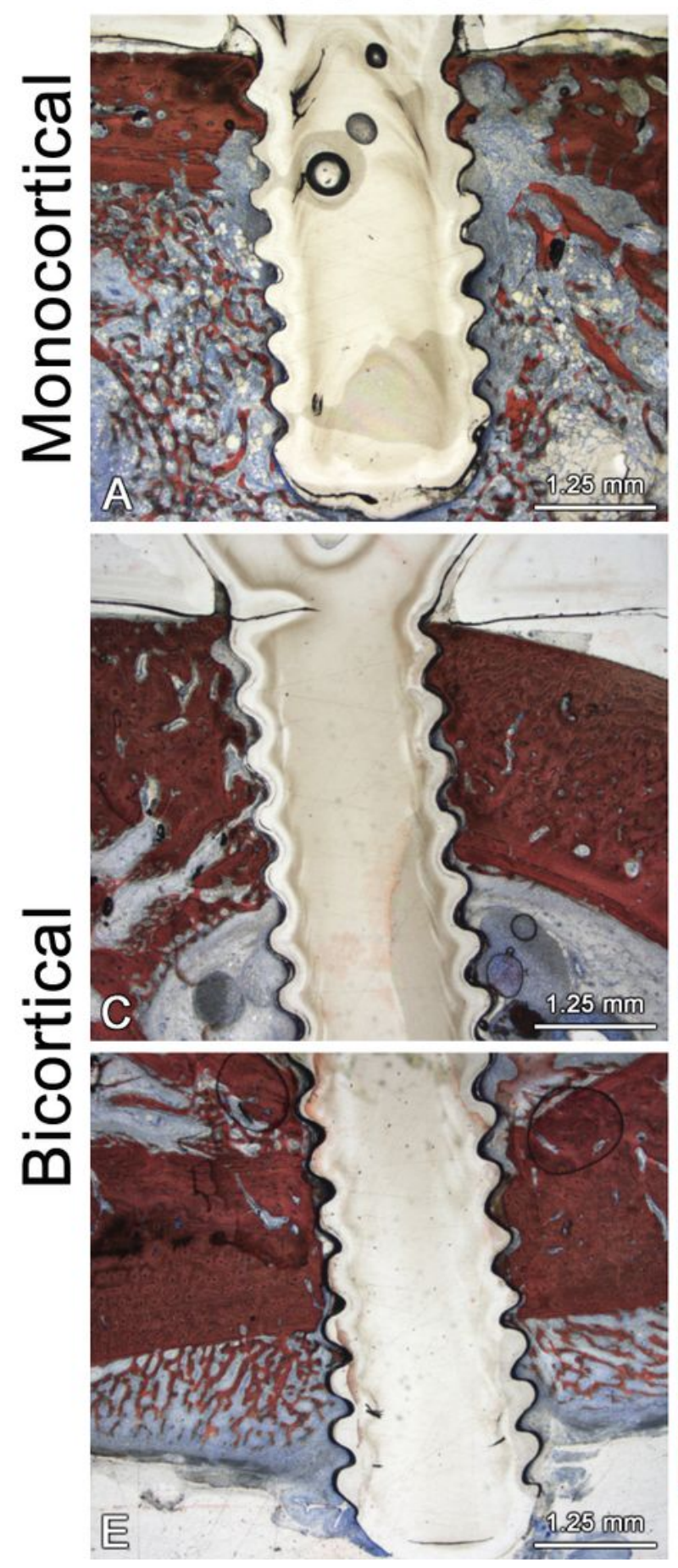

Metallic
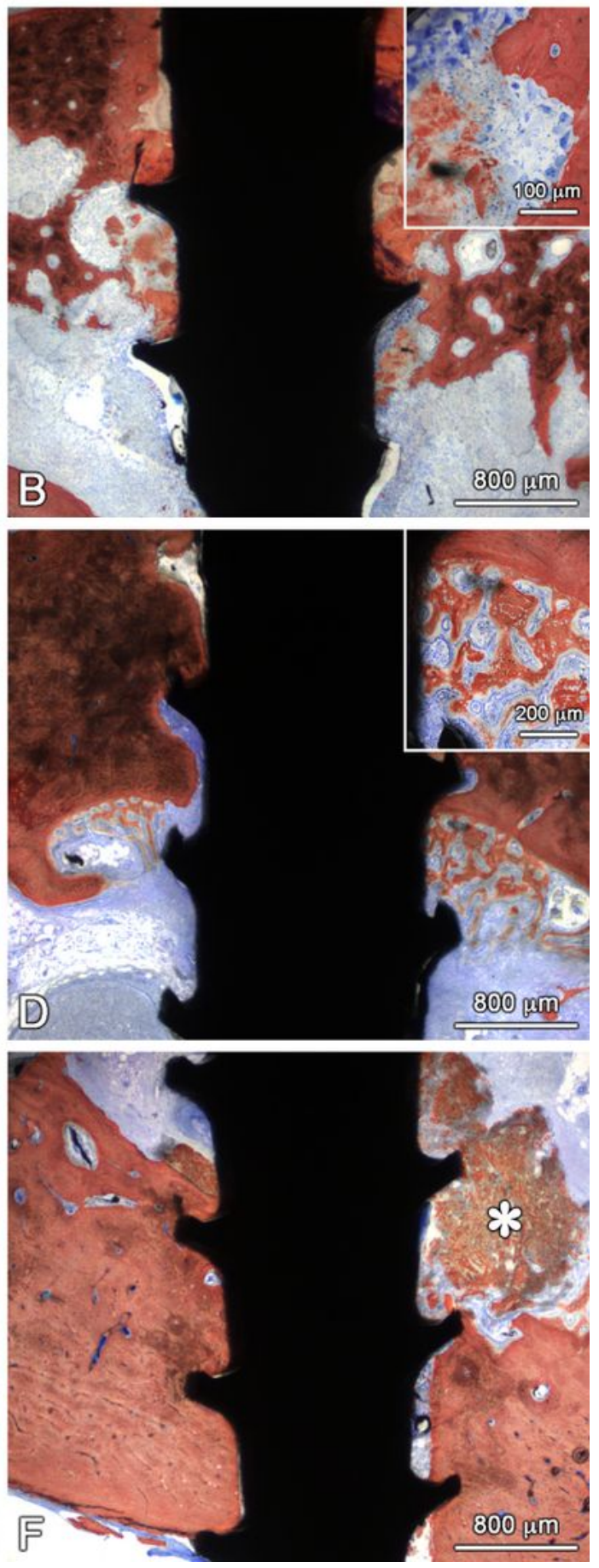

Figure 5

Monocortical absorbable and metallic screws of corresponding miniplates placed in osteotomized dog mandibles at 2 weeks post-placement. (a) While the parent, cortical lamellar bone was in direct contact with the first upper threads of the monocortical absorbable screw, in the bone marrow a newly formed woven bone occasionally interacted with the screw surface. (b) In addition to the presence of parent, lamellar bone juxtaposed to the monocortical metallic screw, some areas between threads were filled with 
amorphous bone debris. Also, intense osteoclast activity could be observed in sites of cancellous lamellar bone near the threads at the bone marrow level (inset). (c, e) The bone contact with absorbable bicortical screw occurred mostly in the buccal and lingual corticals, with the latter showing newly formed woven as periosteal reaction. $(d, f)$ For the bicortical metallic screw, direct bone contact to its surface took place mostly in the buccal and lingual corticals, with areas of newly formed woven bone as endosteal reaction (d inset) and remaining bone debris close to the screw at the inner surface of lingual cortical (asterisk). Alizarin Red and Stevenel blue staining

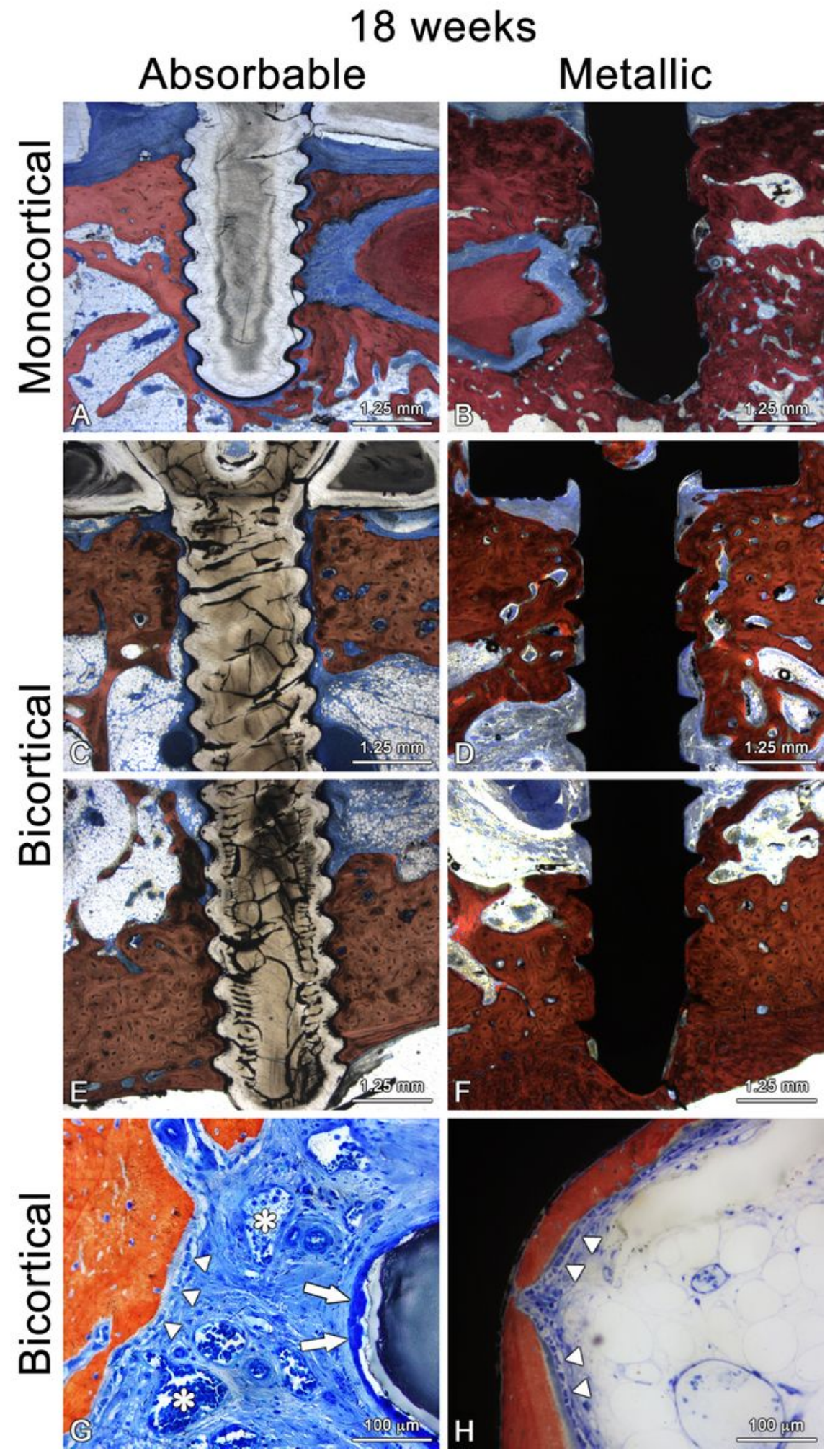




\section{Figure 6}

Monocortical absorbable (a, c,e,g) and metallic (b, d, f, h) screws of corresponding miniplates placed in osteotomized dog mandibles at 18 weeks post-placement. (a) Dense connective tissue was observed between both cortical and spongy bone and the monocortical absorbable screw surface. (b) Lamellar cortical and spongy bone established a direct contact with the monocortical metallic screw surface. (c,e) Although both buccal and lingual corticals were observed in close proximity to the body of bicortical absorbable screws, only the lingual cortical exhibited bone margins in direct contact to the screw surface and a pronounced periosteal reaction. $(d, f)$ The bone margins of both buccal and lingual corticals were in direct contact with the bicortical metallic screw surface, except in a few areas that showed loose connective tissue between them. (g) Higher magnification of the tissue-absorbable screw interface showing highly vascularized (asterisks), dense connective tissue with multidirectional collagen fiber bundles interposed between the screw surface and the bone margins. The irregular, degrading screw surface was lined by multinucleated giant cells (arrows), while bone margins showed osteoblasts (arrowheads) that lined osteoid and occasional osteoclasts on the mineralized matrix. (h) Areas of active contact osteogenesis (arrowheads) were observed in the bone marrow-metallic screw interface. Alizarin Red and Stevenel blue staining 